\title{
Conceptual and perceptual processes in prospective remembering: Differential influence of attentional resources
}

\author{
DEBORAH MCGANN \\ University of Portsmouth, Portsmouth, England \\ JUDI A. ELLIS \\ University of Reading, Reading, England \\ and \\ ALAN MILNE \\ University of Aberdeen, Aberdeen, Scotland
}

\begin{abstract}
Does prospective remembering rely on strategic, attentionally demanding processes? We report three experiments suggesting that the extent to which attentional processes are required varies according to the character of ongoing task processing. Study-test changes in the semantic context of targets had a negative effect on prospective memory performance when participants were engaged in a conceptually focused (sentence verification) task (Experiment 1). Similarly, prospective remembering was lower following study-test changes in perceptual format (font) in the context of a perceptually focused (readability rating) ongoing task (Experiment 2). However, although dividing attention at retrievalhad a negative effect during the performance of an ongoing conceptual task (Experiments 1 and 3 ), it had no effect during an ongoing perceptual task (Experiments 2 and 3). Thus, both perceptual and conceptual process are implicated in prospective remembering, but the processing focus of the task in which remembering should occur may mediate the requirement for strategic processes. These findings suggest that more than one retrieval route is available for prospective remembering and that selection of the route depends on the nature of the task and the processing that occurs at encoding and retrieval.
\end{abstract}

Remembering to water your house plants twice a week or to relay a message to a colleague when you next see $\mathrm{him} / \mathrm{her}$ are examples of prospective memory tasks. Successful completion of these everyday activities entails recalling an intention, which could not be executed immediately, at an appropriate moment in the future, usually when we are involved in some other activity or ongoing task (see, naturally occurring intentions, Ellis, 1988). By contrast, retrospective memory tasks involve remembering what we have done or information we have previously learned, such as recognition of an old school friend or recalling what film we saw yesterday. One of the features that distinguishes prospective from retrospective memory tasks is that prospective tasks do not have obvious external cues (Harris, 1984). This characteristic has led

This work was supported in part by a University of Reading Research Endowment Trust Grant. Experiments 1 and 2 were conducted in partial fulfillment of the requirements for D.M.'s doctorate at the University of Reading, and an interim report of these experiments was presented at the First International Conference on Prospective Memory, Hatfield, England, July 2000. Correspondence concerning this article should be addressed to D. McGann, Division of Psychology, University of Northumbria, Newcastle upon Tyne NE1 8ST, England (e-mail: deborah. mcgann@unn.ac.uk). to debate on the nature of the cognitive processes that lead to the timely retrieval of a prior intention and the extent to which these processes are consciously controlled.

Einstein and McDaniel $(1990,1996)$ have proposed that prospective remembering is relatively spontaneous, referring to participants' subjective reports that intended actions "pop into mind" at the appropriate moment. Craik (1986), however, has argued that prospective remembering is a conscious, controlled process that requires a great deal of self-initiated retrieval. One means of resolving this debate is to explore the role of attention in prospective remembering, on the assumption that controlled processes require attentional resources. If controlled processes are important, performance will be impaired when relatively few attentional resources are available (e.g., in older adults) and when greater demands are made on the limited attentional capacity of working memory (e.g., dual-task paradigms), consistent with Hasher and Zacks's (1979) criteria for automaticity.

These issues have commonly been studied (e.g., Einstein \& McDaniel, 1990; Harris \& Wilkins, 1982) using a laboratory paradigm in which participants are actively engaged in an activity or ongoing task and are asked to perform a particular action in response to an event embedded in that task (event-based task) or at a particular 
time (time-based task). Einstein, McDaniel, Richardson, Guynn, and Cunfer's (1995) findings, for example, suggest that event-based prospective remembering is relatively automatic. Although younger adults performed reliably better than older people on a time-based task, no difference was observed on an event-based one. Timebased tasks may require more self-initiated processes than event-based ones because they require continuous monitoring and are not supported by an environmental event (Einstein \& McDaniel, 1990; but see also Rabbitt, 1996). Under some circumstances, however, event-based tasks do appear to rely on consciously controlled processes. Age-related declines in performance have been reported when, for example, atypical category exemplars are used as targets (Mäntylä, 1994; see also Einstein, Holland, McDaniel, \& Guynn, 1992; Maylor, 1993).

McDaniel, Robinson-Riegler, and Einstein (1998) examined the attentionaldemands of event-based prospective memory by using a divided-attention(DA) ${ }^{1}$ manipulation at retrieval in the context of a conceptual pleasantness rating ongoing task. Their DA task involved listening to a series of random numbers and responding orally whenever three consecutive odd numbers occurred (see Craik, 1982; Jacoby, Woloshyn, \& Kelley, 1989). They found a negative effect of introducing this additional task, suggesting that attentional resources play some role in event-based prospective memory task retrieval. Einstein, Smith, McDaniel, and Shaw (1997) also found negative effects of dividing attention in the context of a conceptual ongoing task using a DA task of detecting 9 s in a series of numbers. When attentional demands were manipulated separately at encoding and retrieval, there was an effect of attention at encoding only for young adults. By contrast, only the older adults revealed a decrement in prospective memory with DA at retrieval, suggesting that age differences in event-based prospective memory tasks depend on the demands of the ongoing activities.

In contrast to the findings reported by Einstein and McDaniel and their colleagues, Otani et al. (1997) found no effect of DA at retrieval on prospective remembering, using a short-term memory ongoing task and a DA task of articulatory suppression. These results, inconsistent with Craik's proposal, support Einstein and McDaniel's $(1990,1996)$ suggestion that prospective memory depends on automatic, spontaneous processes. However, one explanation for discrepancy in the above findings hinges on the different nature of the DA tasks employed (Marsh \& Hicks, 1998). Thus, although McDaniel et al.'s (1998) number detection task probably placed relatively high demands on executive processes, Otani et al.'s articulatory suppression task may have been performed more automatically by the phonological loop (Baddeley, 1986, 1992). Consistent with this argument, Marsh and Hicks (1998) found that DA tasks that placed a high demand on executive resources (e.g., star counting) during the retrieval phase had a negative effect on event-based prospective remembering. In contrast, they found no reliable effects with DA tasks that relied on Baddeley's
$(1986,1992)$ slave systems of working memory (e.g., articulatory suppression).

Further analysis of the role of automatic and controlled processes in prospective remembering should help to distinguish between two theoretical frameworks proposed by Einstein and McDaniel (1996). In their notice-plus search model, an event-based environmental cue automatically induces a feeling of familiarity or "noticing" in the participant that leads to a conscious, attention-demanding search for the significance of that cue (the prospective memory task). Using Einstein and McDaniel's (1990) terminology, this model suggests that attention is important during the retrospective memory component of a prospective memory task (i.e., retrieving the content of the task) and relatively less important for the prospective memory component (i.e., remembering to remember, but see Marsh and Hicks's, 1998, intuition that DA interferes with noticing the target event). In contrast, their simple activation model claims that conscious/controlled processes play no part in retrieval: Automatic noticing of the cue leads to a spontaneous, nonattentionally demanding activation of the previous association between the cue and the intended action.

The aim of the present studies was to examine further the role of automatic and controlled processes in eventbased prospective memory tasks. To gain a broader perspective, we examined the effect of divided attention in the context of both conceptual and perceptual ongoing tasks. Most investigations have required participants to respond to targets while engaged in a conceptually focused ongoing task (e.g., pleasantness rating). This has led to a bias in conceiving prospective memory processes as predominantly conceptual. However, as McDaniel et al. (1998) have noted, perceptually driven processes may be important when perceptually based ongoing tasks are used. McGann, Ellis, and Milne (2003) found evidence to support this view using the transfer-appropriate processing framework (see Blaxton, 1989; Morris, Bransford, \& Franks, 1977). They confirmed findings (e.g., McDaniel et al., 1998) that conceptually driven processes are important in prospective remembering by demonstrating that study-test changes in the meaning of targets had a negative effect on performance in the context of a conceptual ongoing task. They also demonstrated the importance of perceptual processes by showing detrimental effects of study-test changes in the perceptual format of targets when a perceptual ongoing task was used.

The following studies were designed to replicate and extend McGann et al.'s (2003) findings using a DA manipulation at retrieval, the attentionally demanding oddnumber task (Craik, 1982; Jacoby et al., 1989), to investigate the role of attention in prospective memory tasks. First, we investigated whether the dominant processes of a prospective memory task influence the extent to which it demands attentional resources. If attention is important for a particular type of prospective memory processing, a DA task would be expected to impair perfor- 
mance more when these processes are the same at study and test than when they change. Second, we explored the possibility that the processes involved in performing an ongoing task influence the extent to which attention is needed. For example, recent studies using implicit memory tasks suggest that conceptual processes rely on attentional resources, whereas perceptual processes are more automatic in nature (Gabrieli et al., 1995; Mulligan \& Hartman, 1996; Wolters \& Prinsen, 1997) when DA tasks with a high cognitive load are employed (Mulligan, 1997). Therefore, if the attentional demands of prospective remembering are dependent on the nature of ongoing task processes, a resource-demanding DA task will have a negative effect on prospective remembering during a conceptual but not during a perceptual ongoing task.

\section{EXPERIMENT 1}

The aim of Experiment 1 was to examine the extent to which conceptual processes play a role in prospective remembering and to investigate whether these processes demand attention at retrieval. The influence of conceptually driven processes on prospective remembering was examined by comparing performance when the meaning of a target was held constant between study and test with performance when target meaning was altered between these two phases. On the basis of previous research, we expected that a change in target meaning would be detrimental to prospective remembering, thus supporting the influence of conceptual processes in prospective memory retrieval (McDaniel et al., 1998; McGann et al., 2003).

Homonyms with the same graphemic and phonemic structure were used as target events. These words have been used to manipulate meaning in retrospective memory investigations of the role of conceptual processes in explicit (Hunt \& Ellis, 1974; Light \& Carter-Sobell, 1970) and implicit (Lewandowsky, Kirsner, \& Bainbridge, 1989; Masson \& Freedman, 1990; Weldon, 1991) tasks. Light and Carter-Sobell, for example, demonstrated that recognition of targets (e.g., jam) is better when context remains the same at study and test (e.g., study: raspberry jam; recognize: raspberry jam) than when it is changed (e.g., study: raspberry jam; recognize: strawberry jam). In addition, they demonstrated that performance in a different-meaning condition (e.g., study: raspberry jam; recognize: traffic jam) was reliably lower than performance in each of the two same-meaning conditions. The meaning of target words was biased in the present study by embedding them in a sentence validity task at study and test, thereby encouraging participants to focus on the relevant semantic features of these stimuli. All participants were presented with the same meaning of the homonyms at study. However, the context was changed across groups at retrieval to bias either the same meaning or a different meaning from that presented at study.

Processing demands were manipulated at retrieval so that attention was divided between either two or three tasks (prospective memory, sentence validity, and DA or odd-number detection). The DA task was removed to examine prospective memory performance under low-load conditions. For simplicity, the three-task condition is referred to as "divided attention" (DA) and the two-task condition is referred to as "full attention" (FA). It was predicted that if prospective memory depends on attentional resources, performance should be worse in the DA (high-load) than in the FA (low-load) condition.

\section{Method}

Participants and Design. Forty-eight participants took part either for course credit in an introductory psychology course or for a small payment (£4). A $2 \times 2$ factorial design was used with the following unrelated factors: study-test change in meaning (same, different meaning) and attentional demands at retrieval (FA, DA). An additional 12 participants were asked to perform the DA task alone so that a baseline level of performance could be calculated for the digit task. This baseline was used to examine task tradeoffs in attention in this and subsequent experiments.

Materials. Additional information on the materials is reported in McGann et al. (2003).

After piloting eight homographs (chest, lap, post, palm, bat, bank, port, fan), bat, bank, port, and fan were chosen as prospective memory targets. These words conformed to the following criteria: they had similar frequency (11-54 per million; Kučera \& Francis, 1967); each meaning formed a noun; and there were small differences in frequency and relatedness between the two meanings (if known; see Durkin \& Manning, 1989). Furthermore, each word fit easily into a sensible target sentence despite constraints on the structure of these sentences. The mean frequency assigned to each meaning of the target words was piloted in our population of participants and was in broad agreement with values reported by previous researchers (e.g., Durkin \& Manning, 1989; Woolen, Cox, Coahran, Shea, \& Kirby, 1980).

Twelve target sentences were prepared, three for each word, corresponding to sentences seen at study and the two test conditions (same, different meaning). The following constraints were imposed on sentence structure: (1) 16-18 word maximum; (2) target words embedded in the sentences (neither first noun nor final word, with the context for target meaning established at the beginning of each statement); (3) no key words common to a study and target sentence pair; and (4) a sensible statement. In the study condition, the dominant meaning of bank (money) and fan (ventilation) was used and the secondary meaning of port (wine) and bat (sport). ${ }^{2}$ For this phase, sentences were presented in lowercase letters with the target word in capitals. In the same-meaning condition, the sense of items biased at study was presented again at test but in a different sentence structure. In the different-meaning condition, the secondary meaning of bank (river) and fan (supporter) was used, and the dominant meaning of port (harbor) and bat (animal). All words in the sentences at test were presented in uppercase letters.

For the practice session, four nontarget uppercase sentences were prepared with the same constraints as those for the target sentences. Two of these had a nonsensical content and two were sensible. Sixteen sentences were constructed for the encoding phase (10 nonsensical, 6 sensible) and 96 (50 nonsensical, 46 sensible) for the main ongoing task. The distinction between nonsensical and sensible sentences was based on piloting. Two undergraduate students were asked to judge each sentence according to this criterion and to comment on any ambiguities. There was $100 \%$ agreement between the judges; however, three sentences regarded as slightly ambiguous were changed.

Materials for the DA manipulation were the same as those used in Craik's (1982) odd-number task. A total of 542 spoken numbers between 1 and 9 were recorded on a tape player at a rate of 1,400 msec 
per number. Within this series, three random odd numbers were presented consecutively at intervals of between 7 and 14 digits.

Procedure. Each participant was informed that the purpose of the study was to compare the abilities of students and head-injured people to process words and numbers. Tasks were presented on a 7100/66 Power Macintosh computer in a session lasting approximately $30 \mathrm{~min}$.

All participants first received practice on the DA task. They were asked to listen to numbers presented at a rate of $1.5 \mathrm{sec}$ per item and to say "now" out loud whenever they heard three consecutive odd numbers. Fifty-seven digits, including four series of three consecutive odd numbers, were presented on a tape player. Following this, participants were given practice on the sentence validity task, for which they were asked to press the key labeled "yes" (the "Z" key) for sensible sentences and the key labeled "no" (the "/" key) for nonsensical ones. Four sentences, two sensible and two nonsensical, were presented on the computer in a random order across participants for $5 \mathrm{sec}$ with an intersimulus interval (ISI) of $1 \mathrm{sec}$.

Practice on the sentence validity task was followed by instructions for the prospective memory task. Participants were informed that they would be presented with a series of additional sentences to judge and that four of these would contain one word in uppercase letters. They were asked to remember these four words in addition to judging sentence validity, and to press the "enter" key if they saw them again at any stage of the experiment. They were instructed to respond as quickly as possible to target words but also to press the response key if they later realized that a word had appeared earlier, even if some time had elapsed and that word was no longer on the screen. Participants were informed that the latter task would allow the researcher to compare their performance with that of people who experience problems with word identification. The prospective memory task was not mentioned again after this. The prospective memory encoding phase followed these instructions and was administered at the same rate as the practice session. Ten nonsensical and 10 sensible (including target) sentences were presented in a different random order across participants. Target sentences were identical across conditions at encoding and were always presented as items 5, 9, 11, and 15. However, the presentation order of the four specific target words was randomized across these locations on a subject-by-subject basis.

Before the prospective memory task was run, a pilot study was conducted to check that participants could learn the content of the prospective memory task in one trial. Twenty participants were presented with the encoding phase of the prospective memory task in the manner just described. Following filler activities, they were asked first to recall the four target words and then to recognize them from a list of 96 nouns. Results showed a high level of accuracy for both recall $(M=3.6, S D=0.75)$ and recognition $(M=3.85, S D=0.37)$.

Target encoding was followed by two filler activities, designed to encourage a certain degree of prospective memory forgetting and to prevent participants from focusing only on the sentence validity phase. Because participants were told that the target could appear in any task (in fact they were only presented in the sentence validity task), they needed to be prepared to carry out the intention at any stage. Two activities, lasting 5-6 min and using words for stimuli, were employed. The first task was an immediate free recall test of 20 nouns presented for $2 \mathrm{sec}$ each with an ISI of $1 \mathrm{sec}$. A lexical decision task followed in which 73 words and 86 nonwords were presented. Items were presented for up to $1,500 \mathrm{msec}$ with an ISI of $500 \mathrm{msec}$ after a response had been made. If no response was made within 1,500 msec, a blank screen appeared until a response was made and was followed by a 500-msec ISI. Words used in these filler activities were not presented at any other stage of the study.

The sentence validity ongoing task, containing the four prospective memory targets, followed the filler activities at the same rate as before and was administered either with (DA) or without (FA) the odd-number task. The sentence validity task was introduced as another test of word processing, and in the DA condition participants were told that the number and word tasks were equally important.
Targets during this phase were presented with either the same or a different meaning to that presented at study; this was not made explicit to participants, however. One hundred sentences (96 nontarget and 4 target) were divided into 10 blocks, each with a random mix of five sensible and five nonsensical items. Across participants, nontarget sentence presentation was randomized throughout the entire set of sentences and the order of target sentences was randomized with one target appearing in each of Blocks 3, 5, 7, and 9. In the DA condition, the tape recorder was started at the same time as the sentence validity task and participants were presented with 485 digits that included 36 instances of three consecutive odd numbers. This task was presented in exactly the same manner in the DA baseline condition but without the prospective memory and ongoing tasks. At the end of this task, participants were prompted to recall the prospective memory task per se and asked to recall the target words. They were then given a list of 32 nouns of similar frequency (11-54 per million; Kučera \& Francis, 1967) in which the four prospective memory target words were embedded and asked to place a tick beside those that they recognized.

\section{Results and Discussion}

Unless otherwise stated, statistically reliable findings in this article are significant at the .05 significance level.

To examine the effects of conceptual changes and attention on performance, the data presented in Table 1 were subjected to a $2 \times 2$ analy sis of variance (ANOVA) with meaning and attention as unrelated factors. This showed a highly significant effect of meaning $[F(1,44)=$ $\left.7.50, M S_{\mathrm{e}}=.13\right]$, with better performance in the samemeaning condition $(M=0.54)$ than in the differentmeaning condition $(M=0.26)$. In addition, there was a highly significant effect of attentional demands at retrieval $\left[F(1,44)=12.61, M S_{\mathrm{e}}=.13\right]$, with higher performance in the FA $(M=0.58)$ than in the DA $(M=0.22)$ condition. There was no reliable interaction $(F<0.84$, $p>.05)$.

As Table 1 illustrates, the possible effects of meaning and attention manipulations on target word recall and recognition were examined following completion of the prospective memory task. ${ }^{3}$ A $2 \times 2$ ANOVA on proportional target recall showed a reliable interaction between meaning and attentional demands at retrieval $[F(1,44)=$ $\left.4.50, M S_{\mathrm{e}}=.05\right]$. No other differences reached significance (all $F_{\mathrm{s}}<3.31, p \mathrm{~s}>.05$ ). Simple effects analyses showed that in the same-meaning condition performance was reliably higher in the FA $(M=0.88)$ than in the DA $(M=0.60)$ condition $[t(22)=3.34, p=.003]$. No other simple effects were significant $(p s>.05)$. Similarly, a $2 \times 2$ ANOVA on proportional target recognition revealed a reliable interaction between attentional demands at retrieval and meaning $\left[F(1,44)=4.58, M S_{\mathrm{e}}=\right.$ .04]. No other differences reached significance (all $F \mathrm{~s}<$ $1.15, p s>.05$ ). Analysis of simple effects showed that in the same-meaning condition performance was reliably higher in the FA $(M=0.94)$ than in the DA $(M=0.79)$ condition $[t(22)=2.23, p=.05]$, and in the FA condition performance was reliably higher in the same-meaning condition $(M=0.94)$ compared to the different-meaning condition $(M=0.75)[t(22)=2.14, p=.04]$.

In view of differences in target recall between conditions, we computed prospective memory performance as 
Table 1

Experiment 1: Proportion (and Standard Deviation) of Prospective Memory Responses, Target Recall, and Recognition

\begin{tabular}{|c|c|c|c|c|c|c|c|c|}
\hline \multirow[b]{3}{*}{ Measure } & \multicolumn{4}{|c|}{ Full Attention } & \multicolumn{4}{|c|}{ Divided Attention } \\
\hline & \multicolumn{2}{|c|}{$\begin{array}{c}\text { Same } \\
\text { Meaning }\end{array}$} & \multicolumn{2}{|c|}{$\begin{array}{l}\text { Different } \\
\text { Meaning }\end{array}$} & \multicolumn{2}{|c|}{$\begin{array}{c}\text { Same } \\
\text { Meaning }\end{array}$} & \multicolumn{2}{|c|}{$\begin{array}{l}\text { Different } \\
\text { Meaning }\end{array}$} \\
\hline & $\mathrm{P}$ & $S D$ & $\mathrm{P}$ & $S D$ & $\mathrm{P}$ & $S D$ & $\mathrm{P}$ & $S D$ \\
\hline $\begin{array}{l}\text { Prospective memory task } \\
\text { responses }\end{array}$ & .77 & .39 & .40 & .43 & .31 & .34 & .13 & .23 \\
\hline False alarms & \multicolumn{2}{|c|}{0} & \multicolumn{2}{|c|}{2} & \multicolumn{2}{|c|}{4} & \multicolumn{2}{|c|}{2} \\
\hline Free recall of target words & .88 & .20 & .69 & .28 & .60 & .20 & .71 & .26 \\
\hline $\begin{array}{l}\text { Prospective memory conditional } \\
\text { on target recall }\end{array}$ & .79 & .40 & .49 & .48 & .42 & .40 & .24 & .39 \\
\hline Recognition of target words & .94 & .16 & .75 & .26 & .79 & .18 & .85 & .20 \\
\hline $\begin{array}{l}\text { Prospective memory conditional } \\
\text { on target recognition }\end{array}$ & .79 & .40 & .44 & .45 & .37 & .36 & .15 & .31 \\
\hline All 4 targets correct & \multicolumn{2}{|c|}{67} & \multicolumn{2}{|c|}{25} & \multicolumn{2}{|c|}{8} & \multicolumn{2}{|c|}{0} \\
\hline 1-3 targets correct & \multicolumn{2}{|c|}{17} & \multicolumn{2}{|c|}{33} & \multicolumn{2}{|c|}{50} & \multicolumn{2}{|c|}{33} \\
\hline 0 targets correct & \multicolumn{2}{|c|}{17} & \multicolumn{2}{|c|}{42} & \multicolumn{2}{|c|}{42} & \multicolumn{2}{|c|}{67} \\
\hline
\end{tabular}

Note-False alarms represent the total number of occasions on which participants made a prospective memory response to a nontarget word. Where $n=4,1$ participant made three false responses and another made a single false response. Where $n=2,2$ participants each made a single false response. P, proportion of prospective memory responses.

the proportion of targets correctly responded to as a function of subsequent target recall (Table 1). All participants recalled at least one target word, although 5 failed to recall a target word that had elicited a prior prospective memory response; the latter words were excluded from these participants' proportional score. A $2 \times 2$ unrelated ANOVA revealed a similar pattern to that reported for the nonconditional data. Thus, there was a reliable effect of meaning with higher performance in the same $(M=0.61)$ than in the different $(M=0.36)$ conditions $\left[F(1,44)=4.07, M S_{\mathrm{e}}=\right.$ .18]. Similarly, performance was reliably higher in the FA $(M=0.64)$ than in the DA $(M=0.33)$ condition $[F(1,44)=$ 6.68], with no reliable interaction between attention and meaning $(F<1)$. A similar pattern was revealed following a $2 \times 2$ unrelated ANOVA conducted on proportional prospective memory scores as a function of subsequent target recognition. (All participants recognized at least one target word and one failed to recognize one word that had previously elicited a correct prospective memory response.) Thus performance was reliably higher in the same- $(M=0.58)$ than in the different- $(M=0.30)$ meaning condition $\left[F(1,44)=10.67, M S_{\mathrm{e}}=.15\right]$, and in the FA $(M=0.62)$ than in the DA $(M=0.26)$ condition $[F(1,44)=$ 5.29]. The interaction was not reliable $(F<1)$. It is highly unlikely, therefore, that the effects of attention and meaning were a consequence of variations in the retention of target word information.

To examine the possibility of task tradeoffs, a one-way ANOVA was conducted on DA task performance. This showed a reliable effect of experimental condition $\left[F(2,33)=21.67, M S_{\mathrm{e}}=.029\right]$. Tukey tests revealed that performance was reliably better when the DA task was performed alone $(M=0.96)$ relative to the same- $(M=$ $0.53)$ and the different- $(M=0.64)$ meaning conditions $(p s<.05)$. However, there was no reliable difference between the same-meaning and different-meaning condi- tions, indicating that these tasks required equivalent attentional resources.

The effect of changes in target meaning in this study replicates McDaniel et al.'s (1998) findings and supports their claim that conceptually driven processes are important in prospective memory. Further, the fact that the DA task had a negative effect on prospective memory performance suggests that this task does not meet all of the criteria for automaticity proposed by Hasher and Zacks (1979). Instead, these data indicate that conscious, controlled processes play a role in prospective remembering. Interestingly, however, no interaction was observed between attentional demands and meaning. Thus, our findings indicate that conceptual processes are important in prospective remembering but that these operations are independent of attentional resources. However, prospective remembering in the context of a conceptual ongoing task is attentionally demanding at retrieval. Experiment 2 examined whether this pattern of findings is specific to prospective remembering in the context of a conceptual ongoing task or whether a similar picture is revealed using a perceptual ongoing task. This will tell us whether perceptual prospective memory processes, if important, depend on attentional resources and whether prospective remembering is attentionally demanding at retrieval in the context of a perceptual task.

\section{EXPERIMENT 2}

This experiment investigated the role of perceptual processes in prospective memory and their dependence on attentional resources at retrieval by examining whether performance declines when the perceptual format of targets changes between study and test. Format was manipulated by presenting items either in the same or a different font at study and test. 
The effect of changing typography has been mixed in the memory literature (for a review, see Roediger \& McDermott, 1993). However, Graf and Ryan (1990) proposed that one critical factor in obtaining format effects is the extent to which participants focus on relevant aspects of to-be-remembered information at study and test. When target font was the same at study and test, Graf and Ryan (1990, Experiment 3) found superior performance on tasks of word identification and recognition relative to when it was changed. However, this benefit occurred only when participants encoded items in the context of a perceptual readability rating task and not when a conceptual word pleasantness rating task was employed. The ongoing task employed in the present study involved engaging participants on the perceptual task of readability rating. Using the reasoning described in Experiment 1, we predicted that a reliable drop in prospective memory performance with study-test changes in font would indicate the importance of perceptual processes in prospective remembering (McGann et al., 2003). Processing demands were manipulated at retrieval in the same manner as Experiment 1 . As before, it was predicted that performance would be worse in the DA than in the FA condition if prospective remembering, irrespective of ongoing task processing focus, requires attentional resources.

\section{Method}

Participants and Design. Forty-eight participants, all native English speakers, took part for either course credit or a small payment (£4). None had taken part in Experiment 1. They were randomly assigned to one of four conditions in a $2 \times 2$ factorial with the following factors: study-test change in font (same, different font) and attentional demands at retrieval (FA, DA). Within the same-font condition, half the subjects saw Font 1 at study and test and half saw Font 2 during each phase. In the different-font condition, half the subjects saw Font 1 at study and Font 2 at test and the other half saw Font 2 at study and Font 1 at test.

Materials. The words bush, sheep, drum, and honey were used as prospective memory targets. These words were of similar frequency to each other and to the nontarget items used in this experiment (11-54 per million; Kučera \& Francis, 1967). Two versions of the four words, each in uppercase and 40-point type, were prepared for presentation at study and test: one in Font 1 (Macintosh-based Poptics Three) and one in Font 2 (Macintosh-based Ogilvie). These fonts (and others used here) were chosen on the basis of piloting.

For the nontarget items, four words were selected for practice on the readability rating task in the Macintosh-based fonts of Kashmir, Grunge Update, Swing, and Sassy Reg. At encoding, the target words were embedded in 16 words, all in uppercase and of the same size as the targets, in 3 different fonts (5 in Kashmir, 6 in Grunge Update, and 5 in Swing). Targets were embedded among 96 uppercase words at retrieval: 16 nontarget words were presented in the same font as the target (Font 1 or Font 2, depending on condition), 20 in the corresponding target font (Font 1 or Font 2), and 20 in each of the following three fonts: Kashmir, Grunge Update, and Swing. This was designed to make targets appear equally distinctive/ nondistinctive across conditions (see McGann et al., 2003).

The same DA manipulation was used in this study as in Experiment 1 . Because the prospective memory task was slightly shorter, however, 407 random numbers instead of 542 were presented at a rate of $1,400 \mathrm{msec}$ per number.

Procedure. Participants were told that the aim of the study was to investigate responses to fonts and pictures used in advertising.
Stimuli were presented on a 7100/66 Power Macintosh computer in a session lasting approximately $30 \mathrm{~min}$. The first phase involved practice on the DA task, administered as in Experiment 1 and followed by practice in the ongoing task of readability rating, for which participants received written instructions. Four words were presented on the computer individually with a 4-point rating scale underneath each item: 1 (very easy to read), 4 (very difficult to read), for $4 \mathrm{sec}$ each with an ISI of $1 \mathrm{sec}$. Participants were asked to respond by pressing the appropriate (1-4) key. The presentation manner and rate of subsequent readability and DA tasks were identical to those in these practice sessions.

After the practice session, written instructions for the prospective memory task were presented to participants in the same font as the encoding font condition to which they had been assigned (Font 1 or Font 2). They were told that they would be presented with more words to rate for readability, four of which would appear in the same font as the instruction sheet, and that these should be remembered while they were completing the ongoing task. Further, they were informed that they should press the "enter" key if they saw these items again at any later stage of the experiment no matter how they appeared (the latter clause was highlighted with a fluorescent pen). They were asked to respond as quickly as possible and to press the response key immediately if they later realized that a word had appeared at an earlier stage. They were also informed that they might later see other words in the same font as the instruction sheet but should press the key only if the word was one of the original four words. Once these instructions were clear, the prospective memory task was not mentioned again.

Prospective memory instructions were followed by target encoding in the context of a readability rating task that consisted of 20 words, including the 4 targets in Font 1 or Font 2 presented as Items 5, 9, 11, and 15 . Target presentation order was randomized across participants. To maintain the cover story, the 6-min filler activity consisted of answering questions about eight advertisements from popular magazines. This was followed by the prospective memory retrieval phase, during which participants were engaged in the readability rating task. Participants in the DA condition were asked to complete the (equally important) odd-number task at the same time as the readability rating, and each activity was presented in the same manner as the practice session. The readability rating task consisted of 100 randomly presented items (96 nontargets, 4 targets) divided into 10 blocks with targets appearing in Blocks 3, 5, 7, and 9. The DA task was started at the same time as readability rating and consisted of 350 digits with 25 targets. On completion of the prospective memory task, participants were prompted to recall the content of the task, including the 4 target words.

\section{Results and Discussion}

The data, summarized in Table 2, were subjected to a $2 \times 2$ ANOVA with two unrelated factors (font and attention). This revealed a significant effect of font $[F(1,44)=$ $10.85, M S_{\mathrm{e}}=.12$ ], with higher performance in the samefont condition $(M=0.80)$ than in the different-font condition $(M=0.47)$. No other results reached significance $\left(F_{\mathrm{S}}<1.06, p_{\mathrm{S}}>.05\right)$. Once again we examined the possible effects of the experimental variables on target word recall following completion of the prospective memory task. A $2 \times 2$ ANOVA showed that performance was reliably higher in the same-font $(M=0.85)$ than in the differentfont $(M=0.64)$ condition $\left[F(1,44)=4.88, M S_{\mathrm{e}}=.11\right]$ (Table 2). No other effects were reliable (all $F_{\mathrm{S}}<1, p \mathrm{~s}>$ $.05)$. To check that variations in target recall were not responsible for the observed effects on prospective remembering, we computed performance as a function of target 
Table 2

Experiment 2: Proportion (and Standard Deviation) of Prospective Memory Responses and Target Recall

\begin{tabular}{|c|c|c|c|c|c|c|c|c|}
\hline \multirow[b]{3}{*}{ Measure } & \multicolumn{4}{|c|}{ Full Attention } & \multicolumn{4}{|c|}{ Divided Attention } \\
\hline & \multicolumn{2}{|c|}{$\begin{array}{c}\text { Same } \\
\text { Font }\end{array}$} & \multicolumn{2}{|c|}{$\begin{array}{l}\text { Different } \\
\text { Font }\end{array}$} & \multicolumn{2}{|c|}{$\begin{array}{c}\text { Same } \\
\text { Font }\end{array}$} & \multicolumn{2}{|c|}{$\begin{array}{l}\text { Different } \\
\text { Font }\end{array}$} \\
\hline & $\mathrm{P}$ & $\overline{S D}$ & $\mathrm{P}$ & $S D$ & $\mathrm{P}$ & $S D$ & $\mathrm{P}$ & $S D$ \\
\hline Prospective memory task responses & .84 & .31 & .52 & .41 & .75 & .32 & .42 & .36 \\
\hline False alarms & \multicolumn{2}{|c|}{2} & \multicolumn{2}{|c|}{8} & \multicolumn{2}{|c|}{4} & \multicolumn{2}{|c|}{2} \\
\hline Free recall of target words & .83 & .25 & .60 & .43 & .83 & .19 & .67 & .40 \\
\hline $\begin{array}{l}\text { Prospective memory conditional } \\
\text { on target recall }\end{array}$ & .88 & .31 & .57 & .45 & .82 & .31 & .55 & .37 \\
\hline All 4 targets correct & \multicolumn{2}{|c|}{75} & \multicolumn{2}{|c|}{25} & \multicolumn{2}{|c|}{50} & \multicolumn{2}{|c|}{8} \\
\hline 1-3 targets correct & \multicolumn{2}{|c|}{17} & \multicolumn{2}{|c|}{50} & \multicolumn{2}{|c|}{42} & \multicolumn{2}{|c|}{58} \\
\hline 0 targets & \multicolumn{2}{|c|}{8} & \multicolumn{2}{|c|}{25} & \multicolumn{2}{|c|}{8} & \multicolumn{2}{|c|}{33} \\
\hline
\end{tabular}

Note-False alarms represent the total number of occasions on which participants made a prospective memory response to a nontarget word. Where $n=8,1$ participant made three false responses, another made four false responses, and another made a single false response. Where $n=4,2$ participants each made a single false response, and another made two false responses. Where $n=2,2$ participants each made a single false response. $\mathrm{P}$, proportion of prospective memory responses.

recall. Six participants failed to recall any target word (4 in the FA and 2 in the DA, different-target conditions), and therefore their data were excluded from the following analysis. Ten participants failed to recall words that had previously elicited a prospective memory response (3, 3, 3, and 1 in the FA-same, FA-different, DA-same, and DA-different conditions, respectively), and these target words were excluded from the relevant participants' scores. A $2 \times 2$ ANOVA revealed the same pattern as that reported for nonconditional data. Performance was higher in the same- $(M=0.85)$ than the different- $(M=$ $0.56)$ font condition $\left[F(1,39)=6.76, M S_{\mathrm{e}}=.19\right]$. There was no main effect of attention and no interaction between attention and font $\left(F_{\mathrm{S}}<1\right)$. (Unfortunately, target recognition was not assessed in this study.)

To examine possible task tradeoffs, a one-way ANOVA was conducted on DA task performance. This showed a reliable effect of experimental condition $[F(2,33)=29.65$, $\left.M S_{\mathrm{e}}=.01\right]$. Tukey tests revealed that performance was reliably better when the DA task was performed alone $(M=0.96)$ than when it was performed in the same- $(M=$ $0.65)$ and the different- $(M=0.74)$ font conditions. However, there was no reliable difference between the samefont and different-font conditions, indicating that performance on these two tasks required equivalent attentional resources.

The font effect demonstrates the sensitivity of prospective remembering to a perceptual, as well as a conceptual (cf. McDaniel et al., 1998), change in target from study to test. The absence of DA effects at retrieval in this study suggests that conscious recollective processes are not an integral part of prospective remembering. Rather, this effect indicates that retrieval of prospective memory tasks may be relatively automatic in some situations. One possibility is that the perceptually focused context in the present study (data-driven ongoing task with perceptual changes in target information) facilitated a more automatic response. An alternative explanation for the effect of dividing attention on prospective remembering in a conceptual context (Experiment 1) but not in a perceptual one (Experiment 2), however, is that the ongoing tasks used in the two experiments required different amounts of attentional resources. It is possible we found this effect not because of the greater attentional demands of prospective remembering in a conceptual context per se but because the conceptual task used in Experiment 1 was particularly demanding in relation to the perceptual task used in Experiment 2. Indeed, in support of this proposal, performance on the odd-digit task was more impaired in the context of the sentence validity task than in the readability rating task. This hypothesis is examined in Experiment 3.

\section{EXPERIMENT 3}

Experiment 3 examined whether the presentation structure or the processing focus (conceptual or perceptual) was responsible for the effect of dividing attention on prospective remembering found in a conceptual (Experiment 1) but not a perceptual (Experiment 2) ongoing task context.

The manipulation of conceptual and perceptual changes to target items between study and test necessitated the inclusion of specific features for the ongoing tasks used in Experiments 1 and 2. For example, to bias the meaning of target items in Experiment 1, it was necessary to embed them in a sentence context. However, to minimize conceptual processing in the readability rating task (Experiment 2), it was deemed important to present these words as single items. Unfortunately, this meant that the ongoing tasks differed in respects other than their conceptual versus perceptual focus. Furthermore, participants in Experiment $1 \mathrm{knew}$ that their performance on the ongoing sentence validity task could be measured for accuracy, and this may have led to greater (attentiondemanding) anxiety effects relative to Experiment 2, 
which simply required subjective ratings. To overcome these differences, we dropped the study-test change manipulation and compared prospective memory performance on conceptual (pleasantness rating) and perceptual (readability rating) ongoing tasks that pilot studies had confirmed to be equivalent in their attentional demands. Both tasks involved single-item presentation and required subjective judgments. We hypothesized that if presentation structure is important (i.e., embedded vs. single items) in determining the attentional demands of prospective remembering, DA effects would be absent in both the conceptual and the perceptual ongoing task contexts. However, if processing focus (i.e., conceptual vs. perceptual) is a critical factor, there should be a negative effect of DA in the conceptual but not the perceptual task.

\section{Method}

Participants and Design. One hundred and thirty-two native English-speaking undergraduates participated for course credit in an introductory psychology course. Thirty-six participants were used to pilot the attentional demands of the two ongoing tasks (i.e., without the prospective memory task). The other 96 participants took part in the main experiment. A $2 \times 2$ factorial design was used with the following factors: type of ongoing task (readability rating, pleasantness rating) and attentional demands at retrieval (FA, DA).

Materials. As in Experiment 2, the words bush, sheep, drum, and honey were used as prospective memory targets. For the readability rating task, targets were presented in the Macintosh-based font Poptics Three (40 point) at study and test. Words were presented in Times New Roman (20 point) during each phase of the pleasantness rating task. All items were presented in uppercase.

For the nontarget items, four words were selected for practice on the readability rating and pleasantness rating tasks. Participants in the readability rating condition saw these words in the Macintoshbased fonts of Kashmir, Grunge Update, Swing, and Sassy Reg. In the pleasantness rating condition, these words were presented in Times New Roman. As before, all words were presented in uppercase. Nontargets were embedded in the same 96 nontarget uppercase words at retrieval as those used in Experiment 2. For the readability rating task, the same fonts as those in Experiment 2 were used. As before, 16 of the nontarget words were presented in the same font as the target items (Poptics Three, 40 point). In the pleasantness rating task, all words were presented in Times New Roman (20 point).

The same materials were used for the DA manipulation as for the previous experiments.

Pilot work was conducted to assess whether the attentional demands of the pleasantness rating and readability rating tasks were equivalent. Participants were told that the purpose of the study was to examine the effect of word rating on reading and that they would be asked to take part in a number of tasks. These tasks were presented on a Macintosh Performa 630 computer in a session lasting approximately $20 \mathrm{~min}$.

The first phase involved practice on the DA task and was administered in the same manner as Experiments 1 and 2. This was followed by practice on the ongoing task, for which participants received written instructions. In the readability rating task, the practice session was identical to that in Experiment 2. For the pleasantness rating task, four words were presented on the computer individually with a 4-point rating scale beneath each item $(1=$ very pleasant, 4 = very unpleasant $)$, for a fixed presentation of $4 \mathrm{sec}$ each with an ISI of $1 \mathrm{sec}$, and participants were asked to respond by pressing the relevant key. The presentation manner and rate of subsequent rating and DA tasks were identical to those in the practice sessions. After the practice sessions, participants were given a 6-min filler activity consisting of two questionnaires used to pilot another, unrelated, study on attributions and forgetting.

Finally, participants were presented with the DA task either in isolation or with either the readability rating or the pleasantness rating task. Participants who undertook two tasks were told that they had another set of ratings to complete and that while they were doing this they should listen for 3 consecutive odd numbers and say "now" whenever they heard them. They were informed that because each task was equally important they should not concentrate on one more than another. The rating task involved rating 100 words (96 targets plus the 4 target words) for pleasantness or readability. Items were presented in a random order across participants. Those doing the DA task in isolation were simply asked to listen for 3 consecutive odd numbers. They were presented with 350 digits in which there were 25 instances of three consecutive odd numbers. Performance on the DA task was measured in each of the three conditions by calculating the proportion of digit task correct responses out of a maximum score of 25. A one-way ANOVA on these data revealed a reliable effect of condition $\left[F(2,33)=10.95, M S_{\mathrm{e}}=.01\right]$. Tukey tests showed that performance was reliably higher when the DA task was presented alone $(M=0.98)$ than when it was presented with the pleasantness $(M=0.83)$ or readability $(M=0.78)$ rating tasks. However, there was no reliable difference between the readability rating and pleasantness rating tasks, indicating that these tasks required equivalent attentional resources.

Procedure. The main experiment took approximately $30 \mathrm{~min}$ and was identical to the piloting phase up until the end of the practice session on the ongoing task. Following practice on the ongoing task (readability or pleasantness rating), participants were given instructions for the prospective memory task. They were presented with written instructions and told that they would be given four more items to rate. Moreover, while rating these words, they should try to remember them and then press the "return" key on the computer keyboard whenever they saw them again at any other stage of the experiment, whatever their appearance, as described in Experiments 1 and 2. Participants were asked to repeat these instructions and, as previously, the prospective memory instructions were not mentioned again.

Prospective memory encoding followed the instruction phase. Participants were presented with four targets to learn while they rated them for either pleasantness or readability. The words were presented at the same rate as in the practice session in a different random order across participants. Following this, participants were presented with the 6-min filler activity described in the piloting phase. Participants were then provided with the main prospective memory task, presented in the context of a readability rating or pleasantness rating task and either with or without the DA task, depending on experimental condition. The procedure was identical to that used in the pilot phase. For prospective memory target presentation, the full set of 100 items was divided into 10 blocks and targets were presented in a random order, across participants, in Blocks 3, 5, 7, and 9. Nontarget items were randomized across participants. In the DA condition, the tape recorder was started at the same time as the rating task and, as in Experiment 2, participants heard 350 digits with 25 targets. As before, they were told that each task was equally important.

At the end of this task, participants were prompted to recall the prospective memory task per se and the target words. They were then given a list of 100 nouns of similar frequency (11-54 per million; Kučera \& Francis, 1967) in which the 4 prospective memory target words were embedded, and they were asked to place a tick beside those that they recognized.

\section{Results and Discussion}

Again, performance on the prospective memory task was measured by calculating the proportion of correct 
responses. These data, presented in Table 3, were subjected to a $2 \times 2$ ANOVA with two unrelated (task and attention) factors. This revealed a marginally reliable interaction between rating task and attention $[F(1,92)=$ $\left.3.87, M S_{\mathrm{e}}=.11\right]$. No other effects were significant $(F \mathrm{~s}<$ $2.42, p>.05$ ). Analysis of simple effects showed a reliable effect of DA in the pleasantness rating task $[t(46)=$ $-2.45, p=.02]$ but not in the readability rating task $[t(46)=0.23, p>.05]$. The possible effects of rating task and attention manipulations on target word recall and recognition following the prospective memory task were examined. Importantly, as Table 3 illustrates, a $2 \times 2$ ANOVA with two unrelated (task, attention) factors failed to reveal any reliable effects for either recall or recognition (all $F \mathrm{~s}<1.11$ ). It is therefore unlikely that the findings were influenced by individual variations in either target recall or recognition. Nevertheless, for parity, we examined performance as a function of subsequent target recall and target recognition.

A $2 \times 2$ unrelated ANOVA conducted when performance was conditional on target recall failed to reveal any reliable effects of attention, task, or their interaction (all $F \mathrm{~s}<1$ ). The same analysis conducted on performance conditional on target recognition, moreover, revealed only a main effect of attention with higher performance in the FA $(M=0.97)$ than in the DA $(M=0.94)$ condition $\left[F(1,83)=4.38, M S_{\mathrm{e}}=.6\right]$. There was no main effect of task and no interaction (both $F \mathrm{~s}<1$ ). However, as the data in Table 3 reveal, performance conditional on both target recall and target recognition was very high and ceiling effects may be masking possible interactions between attention and task. Moreover, a substantial proportion of participants' data was necessarily excluded from the above analyses. First, 10 participants failed to recall any target word and 9 failed to recognize a single word. Second, 29 participants failed to recall target words that they had previously responded to $(13,8,3,7$, in FA-same, FA-different, DA-same, DA-different conditions, respectively) and 17 failed to recognize such targets $(7,3,2,5$, respectively). This illustrates our earlier cautionary comments on the use of such retrospective data. Not only might performance benefit subsequent recall or recognition, but it might also have a detrimental effect, perhaps mirroring the intention cancelation effect reported by Marsh, Hicks, and Bink (1998), in which information on previously executed actions is rendered inaccessible relative to other information in memory. Most important, however, our analyses failed to reveal any effects of the independent variables on either target recall or target recognition, thus indicating that prospective memory performance is not unduly influenced by these retrospective measures of target retention.

Once again, to examine the possibility of task tradeoffs, a one-way ANOVA was conducted on DA task performance. This showed a reliable effect of experimental condition $\left[F(2,57)=6.54, M S_{\mathrm{e}}=.02\right]$. Tukey tests revealed that performance was reliably better when the DA task was performed alone $(M=0.96)$ than with the pleasantness rating $(M=0.82)$ or the readability rating $(M=$ $0.80)$ task $(p s<.05)$. However there was no reliable difference between the pleasantness rating and readability rating conditions $(p>.05)$. This replicates the findings from the pilot study, showing that the two ongoing tasks have equivalent attentional demands.

Together with the findings of Experiments 1 and 2, these data suggest that the effects of DA on prospective memory retrieval are influenced by the processing required to perform the ongoing task. DA has a reliably negative effect on prospective memory in the context of the conceptually focused tasks of sentence meaning judgment and pleasantness rating but no effect in the context of the perceptually focused task of readability rating.

Table 3

Experiment 3: Proportion (and Standard Deviation) of Prospective Memory Responses, Target Recall, and Recognition

\begin{tabular}{|c|c|c|c|c|c|c|c|c|}
\hline & \multicolumn{4}{|c|}{ Full Attention } & \multicolumn{4}{|c|}{ Divided Attention } \\
\hline & \multicolumn{2}{|c|}{$\begin{array}{l}\text { Pleasantness } \\
\text { Rating } \\
\end{array}$} & \multicolumn{2}{|c|}{$\begin{array}{l}\text { Readability } \\
\text { Rating } \\
\end{array}$} & \multicolumn{2}{|c|}{$\begin{array}{l}\text { Pleasantness } \\
\text { Rating } \\
\end{array}$} & \multicolumn{2}{|c|}{$\begin{array}{c}\text { Readability } \\
\text { Rating }\end{array}$} \\
\hline & $\mathrm{P}$ & $S D$ & $\mathrm{P}$ & $S D$ & $\mathrm{P}$ & $S D$ & $\mathrm{P}$ & $S D$ \\
\hline Prospective memory task responses & .85 & .30 & .72 & .34 & .60 & .40 & .75 & .32 \\
\hline False alarms & \multicolumn{2}{|c|}{3} & \multicolumn{2}{|c|}{5} & \multicolumn{2}{|c|}{5} & \multicolumn{2}{|c|}{9} \\
\hline Free recall of target words & .67 & .34 & .67 & .35 & .60 & .40 & .64 & .29 \\
\hline \multicolumn{9}{|l|}{ Prospective memory conditional } \\
\hline on target recall & .96 & .21 & .98 & .11 & .92 & .17 & .96 & .20 \\
\hline Recognition of target words & .76 & .34 & .70 & .36 & .68 & .39 & .76 & .24 \\
\hline $\begin{array}{l}\text { Prospective memory conditional } \\
\text { on target recognition }\end{array}$ & .95 & .21 & .98 & .11 & .83 & .31 & .89 & .28 \\
\hline All 4 targets & \multicolumn{2}{|c|}{75} & \multicolumn{2}{|c|}{46} & \multicolumn{2}{|c|}{33} & \multicolumn{2}{|c|}{54} \\
\hline $1-3$ targets & \multicolumn{2}{|c|}{17} & \multicolumn{2}{|c|}{46} & \multicolumn{2}{|c|}{46} & \multicolumn{2}{|c|}{42} \\
\hline 0 targets & \multicolumn{2}{|c|}{8} & \multicolumn{2}{|c|}{2} & \multicolumn{2}{|c|}{21} & \multicolumn{2}{|c|}{4} \\
\hline
\end{tabular}

Note-False alarms represent the total number of occasions on which participants made a prospective memory response to a nontarget word. These data were provided by 4 of the 32 participants in the pleasantness rating task and 10 of the 32 participants in the readability rating task. P, proportion of prospective memory responses. 


\section{GENERAL DISCUSSION}

These studies replicate McGann et al.'s (2003) findings in demonstrating that both perceptual and conceptual processes influence prospective remembering when the processes required to perform the ongoing task are compatible with the dimension along which the target changes between study and test. Study-test changes in target meaning have a negative effect on performance in the context of a conceptual task, and study-test changes in the perceptual format of targets (font) have a negative effect on performance when a perceptually focused ongoing task is used. Moreover, the absence of a reliable interaction with the DA manipulation suggests that the perceptual and conceptual processes underlying the effects of encoding-retrieval compatibility are independent of attentional resources.

DA had a negative effect on prospective remembering in the context of conceptual ongoing tasks (Experiments 1 and 3 ) but not perceptual ongoing tasks (Experiments 2 and 3). These findings suggest that the processes required for the ongoing task influence the extent to which attention is needed in prospective remembering. We can be fairly confident that the difference between conceptual and perceptual processes is the key factor because the effect of attention occurs not only in a conceptual task that is different (sentence meaning judgment, Experiment 1) in format from the perceptual task of readability rating (Experiment 2) but also in one that is similar (pleasantness rating, Experiment 3).

The demonstration that attentional resources play a role in prospective remembering, albeit only in the context of a conceptual ongoing task, provides some support for Einstein and McDaniel's (1996) "notice-plus search" model. This states that automatic feelings of familiarity or fluency elicit initial noticing of the prospective memory target, which is then followed by a conscious, controlled search for the significance of that event. Although Einstein and McDaniel's (1996) proposal rests on the assumption that initial noticing depends on perceptual fluency, an elaboration of this view has been proposed by McGann et al. (2003) that is based on Rajaram's (1996; see also Rajaram \& Roediger, 1997) reformulation of the processes underlying recognition memory components. According to this, processing fluency leads to feelings of familiarity when a person is recognizing an event and can be influenced by both perceptual and conceptual processing. The second, the conscious recollection stage is influenced by distinctive (perceptual or conceptual) processing (Rajaram, 1996; Rajaram \& Roediger, 1997). It is therefore possible that study-test matches in conceptual and perceptual features could influence feelings of familiarity (cf. noticing) but that controlled, distinctive processing for the significance of those feelings occurs only in a conceptual processing context. The "simple activation" model, therefore, may provide a better description of the processes involved when prospective remembering occurs in a perceptual context. Under these condi- tions, conscious/controlled processes are absent and automatic noticing of the prospective memory target leads to spontaneous activation of the cue-target association.

This reformulation of the processes underlying recognition also has implications for an alternative account of prospective memory retrieval-automatic associationproposed by McDaniel et al. (1998) and based on Moscovitch's (1994) systems framework. This proposal, a reformulation of the simple activation model, is based on the assumption that event-based prospective memory tasks are similar to direct associative episodic memory tasks. Moscovitch proposed that episodic memory tasks are mediated in part by a memory module that is subserved by components of the hippocampus. When a cue (e.g., a target event) is presented to this module, information associated with it (e.g., an intended action) is delivered to consciousness rapidly and involuntarily in a reflexive manner. McDaniel et al. argue that this model is able to account for their claim that prospective memory is primarily conceptually driven because the memory module presented in this framework is facilitated by semantic encoding, which strengthens the association between a cue (target event) and a memory trace (intended action). However, McDaniel et al. fail to explain the mechanisms by which semantic processing, which is focused only on target information at encoding, strengthens the association between a target and a prospective memory action. Moreover, they do not show how the model explains the present finding that perceptual processing is also important in prospective remembering.

It may be possible to explain the present data by combining Moscovitch's (1994) framework with the basic assumptions of a transfer-appropriate processing approach. Specifically, it is feasible that an association is formed at study between the encoded conceptual and/or perceptual features of the target event and a prospective memory action. If these target features are processed again at retrieval, owing to ongoing task demands, the reflexive action of the proposed memory module presents the target and its associated prospective memory action to consciousness, thereby allowing the intention to be performed. The main drawback of this account, however, is that Moscovitch's associative memory module is assumed to depend on few cognitive resources. Accordingly, it fails to explain the present finding that a DA task at retrieval has a negative effect on performance, at least in the context of a conceptual ongoing task.

Taken together, it would appear that none of these models is able to provide an adequate account of all of the findings reported in this article. We suggest, therefore, that prospective memory retrieval operates through a combination of these routes, and that the specific mechanisms underlying retrieval depend on the characteristics of the prospective memory task and the processing that occurs at encoding and retrieval (see the modified tworoute model of retrieval; Ellis, Milne, \& McGann, 1997). Specifically, all other aspects of prospective memory and ongoing tasks being equal, when these processes are 
primarily perceptual, retrieval may require only one stage of processing; when they are conceptual, the second stage may be necessary. McDaniel and Einstein (2000) have also proposed a multiprocess framework in which they argue that "people can rely either on strategic or relatively automatic processes for prospective memory retrieval and do so in different degrees according to the task" (p. S142). Our data suggest that one aspect of a prospective memory task that influences reliance on strategic processes is the processing focus of the ongoing task in which prospective remembering should occur. Other possible candidates indicated by our data include the degree to which target words are learned. The recall data in these studies indicate relatively low levels of learning; overlearning may reduce reliance on strategic processing at retrieval. It is also possible that prospective remembering is more dependent on strategic processes when target words are embedded in a sentence (see the relative difference in the effects of DA in Experiments 1 and 3); MacLeod (1989) has made a similar point regarding priming in implicit memory.

We have demonstrated that prospective remembering can be either perceptually based or conceptually based and that the attentional demands that it makes vary according to the nature of the processing focus of the ongoing task in which it takes place. The processes that underpin prospective remembering, therefore, appear to be relatively flexible, varying according to different facets of the prospective memory task. Given the diversity of naturally occurring intentions and the contexts in which remembering should occur, this flexibility is perhaps unsurprising. An important task for the future is to identify key variables, in addition to ongoing task processing focus, that influence the requirement for attentional resources and to discover the mechanisms by which they exert this influence.

\section{REFERENCES}

BAdDEley, A. (1986). Working memory. Oxford: Oxford University Press.

BADDELEy, A. (1992). Working memory. Science, 255, 556-559.

Blaxton, T. A. (1989). Investigating dissociations among memory measures: Support for a transfer-appropriate processing framework. Journal of Experimental Psychology: Learning, Memory, \& Cognition, 15, 657-668.

CRAIK, F. I. M. (1982). Selective changes in encoding as a function of reduced processing capacity. In F. Klix, J. Hoffman, \& E. Van der Meer (Eds.), Cognitive research in psychology (pp. 152-161). Berlin: FRG.

CraIK, F. I. M. (1986). A functional account of age differences in memory. In F. Klix \& H. Hagendorf (Eds.), Human memory and cognitive capabilities: Mechanisms and performances (pp. 409-422). Amsterdam: Elsevier.

Durkin, K., \& Manning, J. (1989). Polysemy and the subjective lexicon: Semantic relatedness and the salience of intraword senses. Journal of Psycholinguistic Research, 18, 577-612.

Einstein, G. O., Holland, L. J., McDaniel, M. A., \& Guynn, M. J. (1992). Age-related deficits in prospective memory: The influence of task complexity. Psychology \& Aging, 7, 471-478.

Einstein, G. O., \& McDaniel, M. A. (1990). Normal aging and prospective memory. Journal of Experimental Psychology: Learning, Memory, \& Cognition, 16, 717-726.
Einstein, G. O., \& McDaniel, M. A. (1996). Retrieval processes in prospective memory: Theoretical approaches and some new empirical findings. In M. Brandimonte, G. O. Einstein, \& M. A. McDaniel (Eds.), Prospective memory: Theory and applications (pp. 115-141). Hillsdale, NJ: Erlbaum.

Einstein, G. O., McDaniel, M. A., Richardson, S. L., Guynn, M. J., \& CUNFER, A. R. (1995). Aging and prospective memory: Examining the influences of self-initiated retrieval processes. Journal of Experimental Psychology: Learning, Memory, \& Cognition, 21, 996-1007. Einstein, G. O., Smith, R. E., McDaniel, M. A., \& Shaw, P. (1997). Aging and prospective memory: The influence of increased task demands at encoding and retrieval. Psychology \& Aging, 12, 479-488.

ElLIS, J. A. (1988). Memory for future intentions: Investigating pulses and steps. In M. M. Gruneberg, P. E. Morris, \& R. N. Sykes (Eds.), Practical aspects of memory: Current research and issues (Vol. 1, pp. 371-376). Chichester, U.K.: Wiley.

Ellis, J. A., Milne, A., \& McGann, D. (1997, May). Conceptual and perceptual processes in prospective remembering. Paper presented at the International Workshop on Prospective Memory, Leuven.

Gabrieli, J. D. E., Stone, M. V., Shackleton, K., Thompson-Schill, S. L., Ladd, S. L., Vaidya, C. J., \& Chari, A. (1995, November). Attention and implicit memory for words. Paper presented at the 36th Annual Meeting of the Psychonomic Society, Los Angeles.

Graf, P., \& RYAN, L. (1990). Transfer-appropriate processing for implicit and explicit memory. Journal of Experimental Psychology: Learning, Memory, \& Cognition, 16, 978-992.

Harris, J. E. (1984). Remembering to do things: A forgotten topic. In J. E. Harris \& P. E. Morris (Eds.), Everyday memory, actions and absent-mindedness (pp. 71-92). London: Academic Press.

Harris, J. E., \& Wilkins, A. J. (1982). Remembering to do things: A theoretical framework and an illustrative experiment. Human Learning, 1, 123-136.

HASHER, L., \& ZACKs, R. T. (1979). Automatic and effortful processes in memory. Journal of Experimental Psychology: General, 108, 356-388.

Hunt, R. R., \& Ellis, H. C. (1974). Recognition memory and degree of semantic contextual change. Journal of Experimental Psychology, 103, 1153-1159.

JACoBy, L. L., Woloshyn, V., \& Kelley, C. (1989). Becoming famous without being recognized: Unconscious influences of memory produced by dividing attention. Journal of Experimental Psychology: General, 118, 115-125.

KuČERA, M., \& FrancIS, W. (1967). Computationalanalysis of presentday American English. Providence: Brown University Press.

LeWAndowsky, S., Kirsner, K., \& Bainbridge, V. (1989). Context effects in implicit memory: A sense-specific account. In S. Lewandowsky, J. C. Dunn, \& K. Kirsner (Eds.), Implicit memory: Theoretical issues (pp. 185-198). Hillsdale, NJ: Erlbaum.

Light, L., \& CARTER-Sobell, L. (1970). Effects of changed semantic context on recognition memory. Journal of Verbal Learning \& Verbal Behavior, 9, 1-12.

MACLEOD, C. M. (1989). Word content during initial exposure influences degree of priming in word fragment completion. Journal of Experimental Psychology: Learning, Memory, \& Cognition, 15, 398-406.

MäNTYLÄ, T. (1994). Remembering to remember: Adult age differences in prospective memory. Journals of Gerontology, 49, P276-P282.

MARSH, R. L., \& Hicks, J. L. (1998). Event-based prospective memory and executive control of working memory. Journal of Experimental Psychology: Learning, Memory, \& Cognition, 24, 336-349.

Marsh, R. L., Hicks, J. L., \& BinK, M. L. (1998). Activation of completed, uncompleted, and partially completed intentions. Journal of Experimental Psychology: Learning, Memory, \& Cognition, 24, 350-361.

Masson, M. E. J., \& Freedman, L. (1990). Fluent identification of repeated words. Journal of Experimental Psychology: Learning, Memory, \& Cognition, 16, 355-373.

MAYLOR, E. A. (1993). Aging and forgetting in prospective and retrospective memory tasks. Psychology \& Aging, 3, 420-428.

McDaniel, M. A., \& Einstein, G. O. (2000). Strategic and automatic processes in prospective memory retrieval: A multiprocess framework. Applied Cognitive Psychology, 14, S127-S144.

McDaniel, M. A., Robinson-Riegler, B., \& Einstein, G. O. (1998). 
Prospective remembering: Perceptually driven or conceptually driven processes? Memory \& Cognition, 26, 121-134.

McGann, D., Ellis, J. A, \& Milne, A. (2003). Conceptual and perceptual processing in prospective remembering. European Journal of Cognitive Psychology, 15, 19-41.

Morris, C. D., Bransford, J. P., \& Franks, J. J. (1977). Levels of processing versus transfer-appropriate processing. Journal of Verbal Learning \& Verbal Behavior, 16, 519-533.

Moscovitch, M. (1994). Memory and working with memory: Evaluation of a component process model and comparisons with other models. In D. L. Schacter \& E. Tulving (Eds.), Memory systems (pp. 269310). Cambridge, MA: MIT Press.

Mulligan, N. W. (1997). Attention and implicit memory tests: The effects of varying attentional load on conceptual priming. Memory \& Cognition, 25, 11-17.

Mulligan, N. W., \& Hartman, M. (1996). Divided attention and indirect memory tests. Memory \& Cognition, 24, 453-465.

Otani, H., Landau, J. D., Libkuman, T. M., St. Louis, J. P., Kazen, J. K., \& Throne, G. W. (1997). Prospective memory and divided attention. Memory, 5, 343-360.

RabBitt, P. (1996). Why are studies of "prospective memory" planless? In M. Brandimonte, G. O. Einstein, \& M. A. McDaniel (Eds.), Prospective memory: Theory and applications (pp. 239-248). Mahwah, NJ: Erlbaum.

Rajaram, S. (1996). Perceptual effects on remembering: Recollective processes in picture recognition memory. Journal of Experimental Psychology: Learning, Memory, \& Cognition, 22, 365-377.

Rajaram, S., \& Roediger, H. L., III (1997). Remembering and knowing as states of consciousness during retrieval. In J. D. Cohen \& J. W. Schooler (Eds.), Scientific approaches to the question of consciousness (pp. 213-240). Mahwah, NJ: Erlbaum.

Roediger, H. L., III, \& McDermott, K. B. (1993). Implicit memory in normal human subjects. In F. Boller \& J. Grafman (Eds.), Handbook of neuropsychology (Vol. 8, pp. 63-131). Amsterdam: Elsevier.
WeLDON, M. S. (1991). Mechanisms underlying priming on perceptual tasks. Journal of Experimental Psychology: Learning, Memory, \& Cognition, 17, 526-541.

Wolters, G., \& Prinsen, A. (1997). Full versus divided attention and implicit memory performance. Memory \& Cognition, 25, 764-771.

Woolen, K. A., Cox, S. D., Coahran, M. M., Shea, D. S., \& Kirby, R. F. (1980). Frequency of occurrence and concreteness ratings of homograph meanings. Behavior Research Methods \& Instrumentation, 12, 8-15.

\section{NOTES}

1. In the retrospective literature an additional task is referred to as a "concurrent" one. Because this term has been used by some prospective memory researchers to refer to an ongoing task, for clarity we describe the additional task as a "divided-attention," or DA task.

2. These meanings were not counterbalanced because the present study was originally a development of an earlier experiment (see McGann et al., 2003) in which participants were also presented with a modulated meaning of target items between encoding and retrieval. Counterbalancing the dominance of the target meaning was omitted in that study because some senses of words were difficult to modulate (e.g., a cricket bat could be something used in sport or a weapon, but the animal sense of the word was difficult to modulate).

3. Caution should be used in interpreting the findings from recall and recognition data since it is possible that these dependent variables were contaminated by whether or not participants responded to these items during the prospective memory task. The relevance of these measures is that they can be used to conditionalize prospective memory performance.

(Manuscript received October 20, 1999; revision accepted for publication June 14, 2002.) 